Journal of Fluid Mechanics

http://journals.cambridge.org/FLM

Journal of

Additional services for Journal of Fluid Mechanics:

Email alerts: $\underline{\text { Click here }}$

Subscriptions: Click here

Commercial reprints: $\underline{\text { Click here }}$

Terms of use : $\underline{\text { Click here }}$

\title{
Segregation patterns in gas-fluidized systems
}

\author{
M. A. GILBERTSON and I. EAMES \\ Journal of Fluid Mechanics / Volume 433 / April 2001, pp 347 - 356
}

DOI: null, Published online: 22 June 2001

Link to this article: http://journals.cambridge.org/abstract_S0022112001003950

How to cite this article:

M. A. GILBERTSON and I. EAMES (2001). Segregation patterns in gas-fluidized systems. Journal of Fluid Mechanics, 433, pp 347-356

Request Permissions : $\underline{\text { Click here }}$ 


\title{
Segregation patterns in gas-fluidized systems
}

\author{
By M. A. GILBERTSON${ }^{1}$ AND I. EAMES \\ ${ }^{1}$ Department of Mechanical Engineering, Bristol University, \\ University Walk, Bristol BS8 1TR, UK \\ ${ }^{2}$ Department of Mechanical Engineering, University College London, \\ Torrington Place, London WC1E 7JE, UK
}

(Received 27 November 2000 and in revised form 18 January 2001)

The formation of segregation patterns in initially homogeneous, fluidized, binary mixtures of particles has been studied. The adjustment of the bed depends on the proportions of fine and coarse particles in the mixture and the gas flow rate relative to the minimum fluidization velocities of the two components. The particles are immobile until the gas flow rate is sufficiently large to fluidize the mixture of particles. When the gas flow rate exceeds this critical value, alternating vertical bands of coarse and fine particles form. At a second critical gas velocity this pattern breaks down and the more familiar pattern of a mixed horizontal band on top of a layer of coarse particles forms. A phase diagram, constructed from experimental observations, shows the conditions for which each of these regimes exists. Its structure is explained in terms of the fluidization and consequent mobility of the mixture components. When horizontal bands are present, the thickness of the lower layer of coarse particles decreases with increasing gas flow rate depending on the proportion of fine particles in the bed. This, and its development, can be understood by analogy with the sedimentation of particles through a turbulent fluid. The experiments imply that the efficiency of mixing by the bubbles in the fluidized bed is very much less than that expected from gas bubbles in a liquid.

\section{Introduction}

Natural and industrial granular flows typically consist of particles of a wide range of sizes owing to either the mixing of particles of different origins, the attrition of large particles, or the agglomeration of fine particles. When a mixture of particles is mobilized, either through bulk movement or when its weight is supported by the flow of a fluid (so that the particles are fluidized), the components can separate from one another to give rise to structures within the flow. The formation of these stuctures is in turn likely to modify the flow dynamics. While the formation of segregation patterns in binary mixtures owing to the action of shear or vibration has been extensively studied (see the review of Ottino \& Khakhar 2000) there has been little work on the segregation patterns formed in gas-fluidized systems. A large number of industrial processes employ fluidization, and the degree of segregration can be of fundamental importance to their performance. Fluidization is also increasingly seen to play an important role in environmental flows, such as pyroclastic flows (Wilson 1980) where it is thought that gas fluidization can account for much of their vigour and extent.

This paper reports new observations of the formation of segregation patterns owing to the fluidization of binary mixtures of particles. In particular, vertical banding patterns are observed in addition to the commonly reported horizontal banding. 
To assist in the interpretation of the new observations, the key physical processes relevant to fluidization are briefly discussed in $\S 2$. The new experimental study of the segregation of binary mixtures of particles is described in $\S 3$ before physical models are developed to interpret the new observations.

\section{The physical processes within a segregating fluidized system}

The behaviour of a granular flow depends upon the degree and nature of the interaction between the particles within it. When there is no gas passing through the particles, interparticle friction dominates and the movement of particles is localized; however, when a fluid is passed through the particles, it exerts a drag on them that supports their weight so that interparticle friction is greatly reduced and they can flow like a bulk material. The flow through the bed of particles can be characterized by a Reynolds number, $R e=u_{g} d_{p} \rho_{g} / \epsilon \mu_{g}$, and the force per unit volume on it can be estimated using the Carmen-Kozeny equation when $\operatorname{Re}<10$,

$$
f=\frac{K \mu_{g} u_{g}(1-\epsilon)^{2}}{d_{p}^{2} \epsilon^{3}}
$$

where $u_{g}$ is the superficial gas velocity, $\epsilon$ is the voidage in the bed, $d_{p}$ is the particle diameter, and $K$ is a dimensionless constant. Empirical measurements suggest that for randomly packed spheres $K \sim 150$ (e.g. Rhodes 1998, p. 83). When the drag on the particles is dominated by viscous stresses, the pressure drop increases linearly with gas speed until the whole weight of the bed is supported and the pressure drop is then independent of flow rate. When the bed is in this fluidized state the net weight of the particles is negligible, and the frictional forces between them are small and they are mobile. The pressure drop over the bed will be equal to the weight of particles; therefore, the gas speed at this point, $u_{m f}$, is given by

$$
u_{m f}=\frac{\epsilon^{3}}{1-\epsilon} \frac{\rho_{p} g d_{p}^{2}}{K \mu_{g}} .
$$

The segregation of particles can be defined as the separation of one type of particle from the general population. Segregation is easily induced in mixtures of particles by a wide range of mechanisms such as vibration, which can give rise to the well-known 'Brazil nut effect', and shear, which in an avalanching binary mixture of particles can lead to the generation of a banded structure with large particles overlying fine (Koeppe, Enz \& Kakalios 1998). The mechanism that is of interest here is gas flow through a mixture of particles. When the gas flow rate is sufficient to fluidize some or all of the particles in a bed then they will become mobile and can segregate. In general, this results in fine particles overlying coarse particles, which is the opposite arrangement to that which results from vibration or shear. However, an agent is required to cause the mixed particles to move. In a fluidized bed this can be provided by regions where the local gas flow rate is in excess of that necessary to fluidize the particles, such as regions surrounding bubbles. Rowe et al. (1965) observed that mixing in fluidized beds of single-sized particles larger than about $100 \mu \mathrm{m}$ was nearly wholly caused by the movement of particles in the wake of the bubbles. For finer particles, those for which bubble-free expansion can take place, the bubbles, when they are present, can also cause mixing similar to eddy diffusion. However, in different circumstances the agitation caused by bubbles can also encourage segregation. In a binary mixture of powders, when all the particles are fluidized the finer particles will 


$\begin{array}{cc}\begin{array}{c}\text { Particle } \\ \text { diameter }(\mu \mathrm{m})\end{array} & \begin{array}{c}\text { Minimum fluidization } \\ \text { velocity }\left(\mathrm{cm} \mathrm{s}^{-1}\right)\end{array} \\ 45-90 & 0.8 \\ 190-212 & 4.5 \\ 250-425 & 9.8\end{array}$

TABLE 1. Properties of particles used in the experiments. The ranges of diameters correspond to those that contain $90 \%$ of the particles in a powder. The velocities of minimum fluidization were measured in the planar container. All the particles used were glass and nearly spherical with a density of $2500 \mathrm{~kg} \mathrm{~m}^{-3}$ and supplied by Potters Ballotini.

be drawn to the top of the bed because the gas flow past them will be in excess of that required to support their weight. When the gas flow rate is not sufficiently large to fluidize all the particles, the larger ones will be drawn down to the bottom of the bed by gravity. In either case small particles form flotsam at the top of the bed and large particles form jetsam at the bottom (Wu \& Baeyens 1998).

Segregation has also been observed in systems where no bubbles are present such as liquid-fluidized beds (Epstein \& Pruden 1999) and gas-fluidized beds of fine particles (Rasul, Rudolph \& Carsky 1999). In these situations, the term 'layer inversion' is often used to describe how in certain situations, as the fluid speed is increased, an initially segregated bed can become mixed and then segregate again, but with the opposite configuration. This behaviour is dependent upon the difference in bulk densities of the component particles compared with the density difference between the particles and fluid (Epstein \& Pruden 1999).

Much of the work on segregation in fluidized beds has taken a one-dimensional approach where particles are either well-mixed or segregated (i.e. whether the particles of one of its components are nearly all found in one region within the bed). Previous research has concentrated on finding a measure for the degree to which particles are mixed, the relationship between the degree of mixture and experimental conditions, especially fluid velocity, and the velocity of minimum fluidization for particle mixtures (Wu \& Baeyens 1998). However, little has been reported on the different structures that form in fluidized beds depending on the conditions in which they are being operated.

\section{Segregation patterns in static fluidized beds}

\subsection{Experimental apparatus}

The fluidized beds were contained within Perspex containers with floors consisting of porous sintered plastic sheeting $(1 \mathrm{~cm}$ thick Vyon D sheeting from Porvair Ltd) through which a gas flow could be passed. The results discussed below come primarily from a planar container $29.1 \mathrm{~cm}$ wide with walls separated by $0.6 \mathrm{~cm}$; however, to ensure that the structures seen were not determined by this particular geometry, they were repeated in a cylindrical container of diameter $29 \mathrm{~cm}$. The properties of the particles are shown in table 1 . The different sets of particles reflect light to different degrees so that the fine particles are distinctly paler than the large particles. This allowed the behaviour of the particle mixtures to be recorded on videotape and measurements to be made directly from the images. The particles were initially mixed well by passing gas through them at a rate far exceeding the speed of minimum 


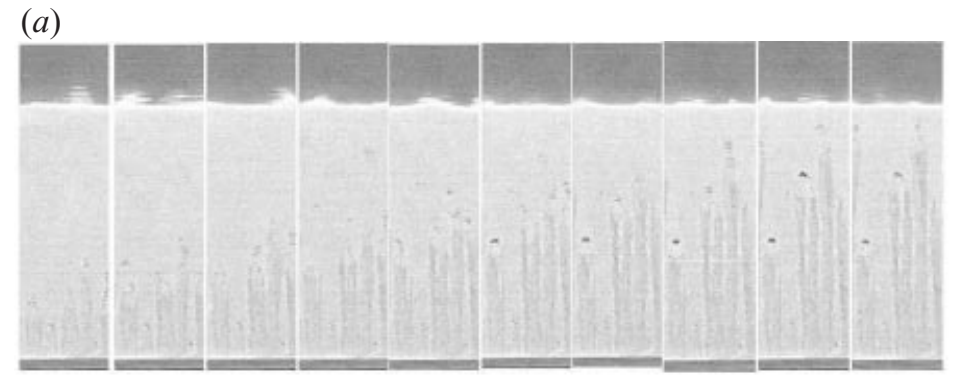

(b)

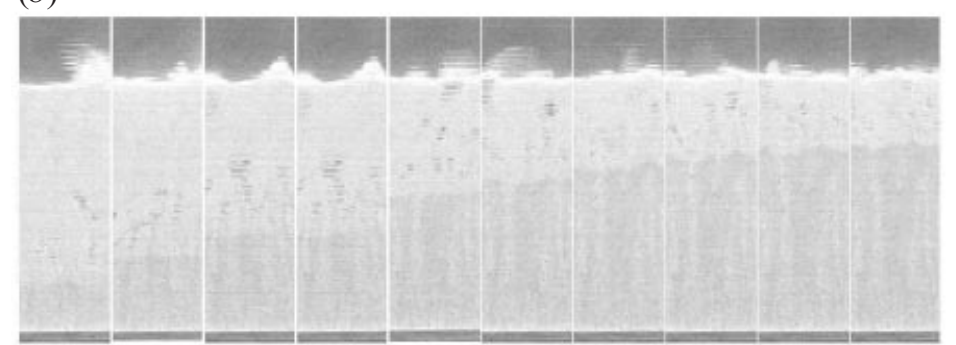

FIGURE 1. Time sequence showing the formation of segregation patterns by gas flowing through an initially homogeneous mixture of particles with equal proportions of $45-90 \mu \mathrm{m}$ and $250-425 \mu \mathrm{m}$ particles. The initial bed height is $12.0 \mathrm{~cm}$. In $(a)$ the gas flow rate is $1.9 \mathrm{~cm} \mathrm{~s}^{-1}$ and the time difference between each image is $1.0 \mathrm{~s}$, and in $(b)$ the gas flow rate is $3.88 \mathrm{~cm} \mathrm{~s}^{-1}$ and the time difference is $2.0 \mathrm{~s}$.

fluidization for either of the constituents of the mixture, and then rapidly turning the gas flow off.

\subsection{Experimental observations}

The gas flow rate was quickly increased from zero to a fixed value and the development of the flow structures was observed. These are illustrated in figure 1 with images of experiments undertaken with a powder consisting of equal proportions by volume of fine and coarse particles.

A variety of different structures can be observed when gas is passed through a given mixture of particles, depending upon the flow rate. At low flow rates, the particles are not mobile and no movement is observed. This state exists until a critical gas velocity is reached when the mixture of particles is fluidized. As the gas flow rate is increased further, trunk-like structures form and the particles segregate into vertical bands, as shown in figure $1(a)$. The bands associated with $90 \mu \mathrm{m}$ and $250-425 \mu \mathrm{m}$ mixtures were observed to be typically just under $1 \mathrm{~cm}$ wide. The bands formed a moving front from the bottom of the bed upwards. Streams of bubbles formed at the top of the vertical bands of coarser particles. The growth of the bands is proportional to time, as shown in figure $2(a)$.

As the gas flow rate is increased further, the vertical bands appeared less definite and partial segregation in the vertical direction occurs so that the coarse particles begin to collect at the bottom of the bed. At higher gas flow rates, full segregation of the bed into horizontal layers was observed, as shown in figure 1(b). The development of the segregation patterns over time is shown in figure $2(a)$ for a number of gas flow rates. It can be seen that the rate of growth of the bottom layer is independent of gas velocity, and so the segregation does not depend on the convective motion of the mixed layer above the layer of coarse particles, which is set by $u_{g}$. 

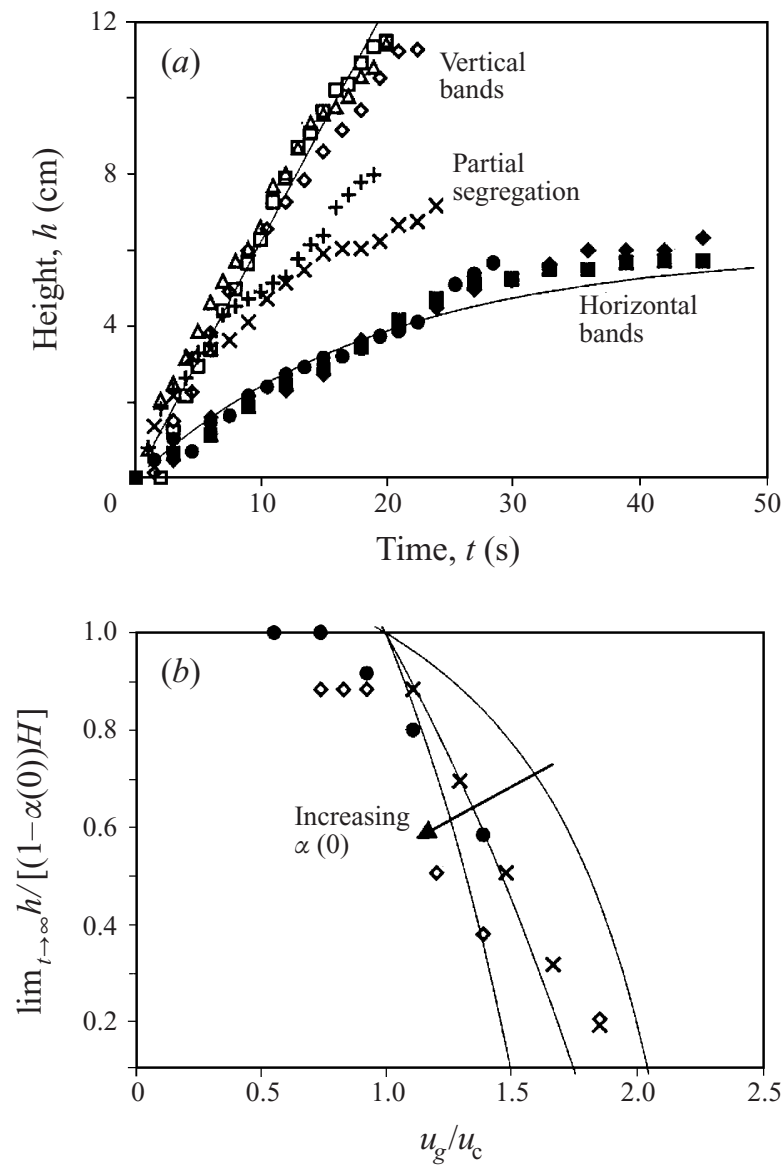

FIGURE 2. Diagrams describing the growth of the segregation structures for a mixture consisting of equal proportions of $45-90 \mu \mathrm{m}$ and $250-425 \mu \mathrm{m}$ diameter particles. $(a)$ Their development with time. The points are for experimental measurements with gas velocities: $\diamond 1.4 \mathrm{~cm} \mathrm{~s}^{-1} ; \square 2.4 \mathrm{~cm} \mathrm{~s}^{-1}$; $\triangle 2.9 \mathrm{~cm} \mathrm{~s}^{-1} ;+3.8 \mathrm{~cm} \mathrm{~s}^{-1} ; * 4.8 \mathrm{~cm} \mathrm{~s}^{-1} ; \bullet 5.8 \mathrm{~cm} \mathrm{~s}^{-1} ; 6.7 \mathrm{~cm} \mathrm{~s}^{-1} ; 7.7 \mathrm{~cm} \mathrm{~s}^{-1}$. The solid lines are the predictions based on (3.10) and (3.7). Similar results are also obtained when the initial fraction of fine particles in the bed $\alpha(0)=0.33$ and 0.60. $(b)$ The ultimate thickness, $h$, of the lower layer of a segregated fluidized bed compared with its thickness if the coarse particles were wholly segregated from the fine particles ( $H$ is the overall depth of the bed). The points are experimental measurements $(\times, \alpha(0)=0.33 ; \bullet, \alpha(0)=0.50 ; \diamond, \alpha(0)=0.66)$, and the curves represent the predictions.

The height of the segregated layer tends asymptotically with time to the height corresponding to the initial fraction of coarse particles in the bed. Figure $2(b)$ shows the ultimate thickness of the lower layer compared with the proportion of large particles in the bed. Below the point of minimum fluidization for the coarse particles, $u_{c}$, all the larger particles sediment out of the mixture into the lower layer. Increasing the gas flow rate results in coarse particles being carried from the lower layer, where they predominate, into the increasingly mixed upper layer. At about twice $u_{c}$ the lower layer is nearly eliminated and the entire bed becomes mixed.

The gas speeds at which transition from one structure to another took place depended on the initial proportions of fine and coarse particles in the bed. From observation of the segregation patterns in a bed that result from different gas flow rates and ratios of volume of fine to coarse particles, a phase diagram for the bed 


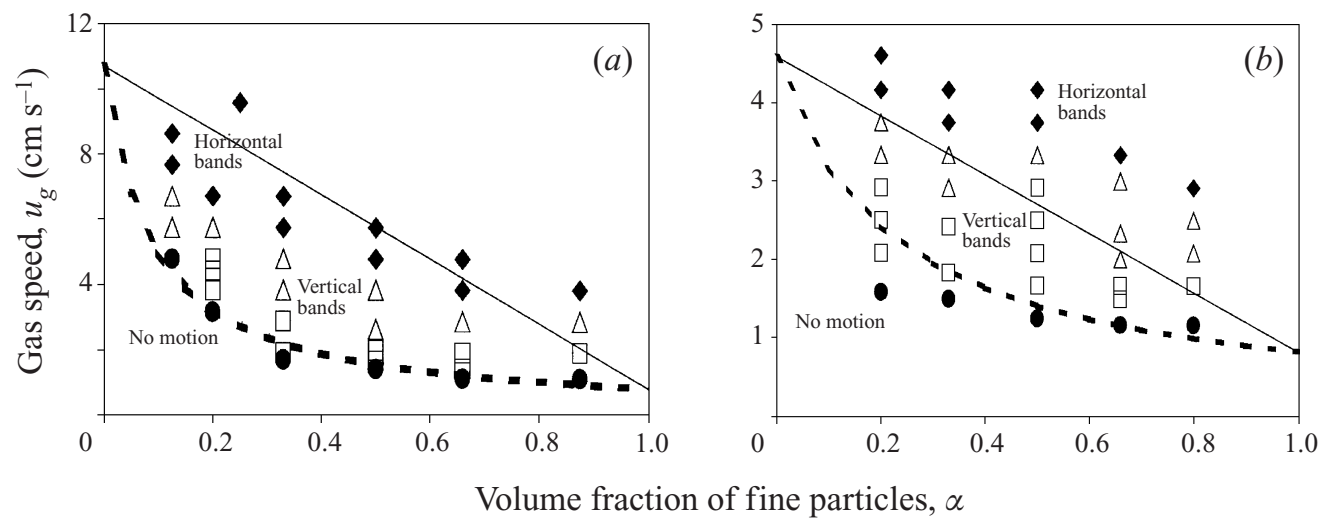

FIGURE 3. Phase diagrams for the mixtures of particles based on experimental observations. The different symbols correspond to the different structures seen: incipient motion $(\bullet)$; vertical bands $(\square)$; partial segregation $(\triangle)$; horizontal banding $(\diamond)$. (a) A mixture of $45-90 \mu \mathrm{m}$ particles and $250-425 \mu \mathrm{m}$ particles, and $(b)$ a mixture of $45-90 \mu \mathrm{m}$ particles and $190-212 \mu \mathrm{m}$ particles.

structure was constructed. Examples are shown in figure 3 for two binary mixtures. The proportion by volume of fine particles in the particle mixture is denoted $\alpha$ and is independent of the voidage in the bed, $\epsilon$. The regimes identified are described as incipient motion, vertical banding, partial segregation (where the vertical bands still exist, but the coarse particles are starting to accumulate at the bottom of the bed), horizontal banding and completely mixed. The experiments were carried out for two different particle mixtures, and the same structures and behaviour were observed. This was also true for experiments performed in the cylindrical container, indicating that the segregation behaviour is not an artefact of the planar geometry, though it was not possible to observe the interior of the bed.

\subsection{Discussion}

\subsubsection{The formation of vertical bands}

The transition from one structure to another in the fluidized bed can be explained by considering the drag forces exerted on the two components in the bed by the gas flow, and the effect that this has on their mobility.

Segregation can only take place when the particles can be mobile, that is when the initial mixture of particles becomes fluidized. This will take place at a gas speed, $u_{g}=u_{b i}$, when the viscous drag exerted by the gas on the two components of the bed balances their total weight. The total drag on the bed can be estimated as a linear combination of the drag force acting on the fine and coarse particles $\left(f_{f}\right.$ and $f_{c}$ respectively). Therefore, when the bed is fluidized,

$$
f_{f}+f_{c}=(1-\epsilon)\left(\rho_{p}-\rho_{g}\right) g .
$$

It is assumed that $\epsilon$ is uniform within the bed. The Carmen-Kozeny equation, (2.1), cannot be strictly applied to mixtures of particles. In order to gain insight into the conditions under which the bed becomes fluidized, the drag on the different fractions of the bed are estimated by applying (2.1) to the mixture for each of its components. Under this approximation and using (2.2), (3.1) reduces to

$$
\alpha \frac{u_{b i}}{u_{f}}+(1-\alpha) \frac{u_{b i}}{u_{c}}=1,
$$


where $u_{f}$ and $u_{c}$ are the velocities of minimum fluidization of the fine and coarse particles respectively. Hence

$$
\frac{u_{b i}}{u_{f}}=\frac{1}{\alpha+(1-\alpha) u_{f} / u_{c}} .
$$

This criterion is shown by the dashed line in figure 3 , and agreement with experimental observations is quite good.

Above $u_{b i}$ the patterns of vertical bands seen in figure 1(a) form. The light bands mostly consist of fine particles, and alternate with dark bands where the coarse particles are concentrated. From figure $1(b)$ it can be seen that a large proportion of the coarse particles in the bed are present within the dark bands. In mixtures with a low concentration of coarse particles, the bands terminate part way up the bed. The bands form because the permeability of the coarse particles is higher than that of the fine particles. This encourages the gas to flow preferentially through regions where the coarse particles are concentrated, locally fluidizing the fine particles and inducing local segregation. The augmented flow manifests itself by streams of bubbles from the tips of the bands, above which further segregation causes the vertical bands of coarse particles to increase in height. Similar structures can be seen in beds which apparently mainly consist of cohesive particles (Wilson 1980); however, empty channels form in beds consisting exclusively of cohesive particles, and it is probably the cohesive nature of the bed that is dominating its behaviour. Wilson (1984) reported the formation of a single channel by a central stream of bubbles in beds of ballotini with a wide size distribution. It is not clear whether the size distribution chosen resulted in a significant proportion of the bed consisting of sufficiently small particles for cohesion to again dominate.

The vertical banded structure will break down when the columns of coarse particles are no longer supported by the surrounding mixture of mostly fine particles when it becomes fluidized. The gas flow rate at which this takes place may be estimated by considering the gas flow through vertical bands of fine and coarse particles. When the voidage of the bed is uniform and the interstitial gas flow is characterized by a low Reynolds number, then from (2.1) it can be seen that the pressure drop across the bands is proportional to the gas flow rate through them. The constants of proportionality through the fine and coarse particles are denoted by permeabilities $\kappa_{f}$ and $\kappa_{c}$ respectively. The gas speeds through the vertical bands of fine and coarse particles are $u_{g f}$ and $u_{g c}$ respectively, and can be related to the total gas flow rate by

$$
(1-\alpha) u_{g c}+\alpha u_{g f}=u_{g},
$$

where $\alpha$ is the volume fraction of fine particles. Since the pressure field must be the same over each horizontal section,

$$
\frac{u_{g f}}{\kappa_{f}}=\frac{u_{g c}}{\kappa_{c}} .
$$

The gas flow rate through the fine particles is thus

$$
\frac{u_{g}}{u_{g f}}=(1-\alpha) \frac{\kappa_{c}}{\kappa_{f}}+\alpha .
$$

The vertical bands are expected to break down when $u_{g f}=u_{f}$. The variation with $\alpha$ of the critical overall gas speed corresponding to this condition is shown by the solid curves in figure 3. The criterion is in reasonable agreement with the experimental 
results, though there is some variation, probably because the bands of material may not be wholly composed of one size or the other.

\subsubsection{The rate of growth of structures in the bed}

Both the vertical bands and the horizontal layers of particles grow with time, as shown in figure 2(a) for an initially homogeneous mixture with equal proportions of fine and coarse particles. The growth of the layer gives a bulk measurement of the rate of segregation, which gives insight into the microscale physics of the bed. This can be exploited by considering the motion of the coarse particles falling at a velocity of $v_{T}$ through the upper mixed layer towards the lower region of the bed where the structures are forming.

When vertical bands are forming and it is assumed that the average fraction of fine particles is independent of height in the bed and is equal to $\alpha(0)$, the initial fraction of fine particles within the bed, the rate of growth of the dark vertical bands is equal to $v_{T} /(1-\alpha(0))$ so that

$$
h=\frac{v_{T} t}{1-\alpha(0)} .
$$

At higher gas velocities when the bed consists of horizontal layers of particles, but the coarse particles are not fluidized, then an analogy with the sedimentation of dense particles from a turbulent convecting layer may be used, where the rate of sedimentation is independent of the turbulent structure (for instance, see Bonnecaze, Huppert \& Lister 1993). The concentration of coarse particles $(1-\alpha)$ in the upper layer then decreases at a rate

$$
\frac{\mathrm{d}(1-\alpha)}{\mathrm{d} t}=-v_{T} \frac{(1-\alpha)}{H-h},
$$

where $h$ and $H$ are the depth of the lower layer of coarse particles and the overall depth of the bed respectively. From figure 2(a), the rate of increase in the thickness of the lower layer is independent of gas flow rate and consequently so must be $v_{T}$. In addition, the thickness of the band of coarse particles is related to the concentration of fines in the upper layer through

$$
\alpha(H-h)=\alpha(0) H .
$$

Combining (3.8) and (3.9), the height of the layer of coarse particles is

$$
\frac{h}{(1-\alpha(0)) H}=1-\exp \left(-\frac{v_{T} t}{\alpha(0) H}\right) .
$$

Thus, the thickness of the coarse particle layer tends asymptotically to $(1-\alpha(0)) H$ over a characteristic time $\alpha(0) H / v_{T}$.

Figure 2(a) shows a comparison between the model predictions and experimental measurements for the development with time of the height of the segregated layer for $\alpha(0)=0.5$. The equivalent fall velocity was determined to be $v_{T}=0.32 \mathrm{~cm} \mathrm{~s}^{-1}$ by fitting the curves. This single fitted parameter allows both models to agree well with the experimental data. The results confirm that the process of segregation is independent of gas flow rate providing that the fine material is fluidized and the coarse particles are not. 


\subsubsection{The transport of particles in a stratified bed}

The presence of a lower layer of coarse particles persists at gas velocities in excess of $u_{c}$ when all the particles within the bed are fluidized and the larger particles are clearly mobile. The thickness, $h$, of the lower layer of coarse particles decreases with increasing gas velocity as shown in figure $2(b)$ as more of them are transported into the upper layer. The value of $h$ is not dependent on the gas flow rate being increased progressively, so there is a balance between the processes, especially vigorous bubbling, that entrain the coarse particles into the mixed upper layer, and those returning the large particles back to the lower layer. It is possible to consider the transport of coarse particles from the lower layer using the same approach as used for considering the transport of fluid by gas bubbles in liquids (Bush \& Eames 1998). The volume flux associated with the bubbles generated in the coarse layer is proportional to $\left(u_{g}-u_{c}\right)$; thus, the rate at which the concentration of coarse particles is increased is $\gamma\left(u_{g}-u_{c}\right) /(H-h) . \gamma$ is a constant that characterizes the volume of coarse particles transported upward by the passage of a single bubble; for gas bubbles in a liquid it is the ratio of the drift volume of the bubble to the bubble volume. The thickness $h$ of the coarse particle layer can be estimated by substituting the source term, $\gamma\left(u_{g}-u_{c}\right) /(H-h)$, into (3.8) and calculating the steady state when $\mathrm{d} \alpha / \mathrm{d} t=0$ applies. Figure 2(b) shows a comparison with the experimental measurements of the thickness of the layer of coarse particles and model predictions. From the best fit with the experimental measurements $\gamma=0.02$. The variation of the thickness of the coarse layer with $\alpha(0)$ and $u_{g}$ appears to be captured by the physical model proposed. The constant $\gamma$ is a measure of the efficiency of the bubbles in mixing the coarse material and suggests that the individual bubbles transport $2 \%$ of the equivalent bubble volume of coarse particles into the upper convecting layer. It is interesting to note that this is significantly smaller than the value of $O(100 \%)$ measured experimentally for high Reynolds number bubbles rising in a thin gap geometry in a liquid by Bush \& Eames (1998).

\section{Conclusion}

New observations of the formation of segregration patterns in fluidized binary systems have been reported and physical models of their development and form are described. It has been shown that a bed consisting of a mixture of particles of different sizes can have a variety of different structures depending on the gas flow rate through it. These structures arise when one or both of the components of the mixture become fluidized so that their weight is supported. This greatly reduces the friction between the particles and thus allows them to become mobile. The different structures occur because they cause the flow through the bed to be heterogeneous and the effect it has on the particles differs according to the local proportions of each component of the mixture. Segregation can persist when the gas flow rate is sufficiently large to fluidize the entire bed. Under such conditions it can be shown that the segregation behaviour can be successfully modelled by drawing an analogy with the sedimentation of particles from a turbulent flow field. The experimental results suggest that the efficiency of mixing by the bubbles in a fluidized bed is very much less than for gas bubbles in a liquid.

I.E. gratefully acknowledges the support of the EPSRC through an Advanced Fellowship at University College London. Equipment support by the Royal Society is gratefully acknowledged. 


\section{REFERENCES}

Bonnecaze, R. T., Huppert, H. E. \& Lister, J. R. 1993 Particle-driven gravity currents. J. Fluid Mech. 250, 339-369.

Bush, J. \& EAMES, I. 1998 Fluid displacement by bubbles rising in a thin gap. Intl J. Multiphase Flow 24, 411-430.

EPSTEIN, N. \& PRUDEN, B. 1999 Liquid fluidisation of binary particle mixtures - III stratification by size and related topics. Chem. Engng Sci. 54, 401-415.

Koeppe, J., EnZ, M. \& KaKalios, J. 1998 Phase diagram for avalanche stratification of granular material. Phys. Rev. E 58, 4104-4107.

Ottino, J. M. \& Khakhar, D. V. 2000 Mixing and segregation of granular materials. Ann. Rev. Fluid Mech. 32, 55-91.

Rasul, M., RudolPh, V. \& CARSKy, M. 1999 Segregation potential in binary gas fluidized beds. Powder Tech. 103, 175-181.

Rhodes, M. 1998 Introduction to Particle Technology. Wiley.

Rowe, P., Partridge, B., Cheney, A., Henwood, G. \& Lyall, E. 1965 The mechanisms of solids mixing in fluidised beds. Trans. Inst. Chem. Engrs 43, 271-286.

WiLson, C. 1980 The role of fluidization in the emplacement of pyroclastic flows: an experimental approach. J. Volcan. Geothermal Res. 8, 231-249.

Wilson, C. 1984 The role of fluidization in the emplacement of pyroclastic flows, 2: experimental results and their interpretation. J. Volcan. Geothermal Res. 20, 55-84.

Wu, S. \& BaEYens, J. 1998 Segregation by size difference in gas fluidized beds. Powder Tech. 98, $139-150$ 